\title{
Screening for hearing loss in early childhood programs
}

\author{
William D. Eiserman $^{\mathrm{a}, *}$, Lenore Shisler ${ }^{\mathrm{a}}$, Terry Foust ${ }^{\mathrm{b}}$, Jan Buhrmann $^{\mathrm{c}}$, \\ Randi Winston $^{\mathrm{d}}$, Karl R. White ${ }^{\mathrm{a}}$ \\ a National Center for Hearing Assessment and Management, 2880 Old Main Hill, \\ Utah State University, Logan, Utah 84322, USA \\ ${ }^{\mathrm{b}}$ Intermountain Health Care, USA \\ ${ }^{\mathrm{c}}$ Illinois College, USA \\ ${ }^{\mathrm{d}}$ Ear Foundation of Arizona, USA
}

\begin{abstract}
This study assessed the feasibility of doing hearing screening in Migrant, American Indian and Early Head Start programs using otoacoustic emissions (OAE) technology. Staff members were trained to screen 0-3-year-old children for hearing loss using handheld OAE equipment and a multi-step screening and referral protocol. Of the 3486 children screened as a part of the study, $77 \%$ passed an OAE screening at the first step, $18 \%$ more passed an OAE screening at the second step, and 5\% were ultimately referred for medical or audiological follow-up. Eighty children were identified as having a hearing loss or disorder of the outer, middle or inner ear requiring treatment. Of these 80 , six had permanent bilateral or unilateral hearing loss. Although the protocol suggested that the multi-step screening procedure should be completed within a 4-week time period or less, analysis of the data showed that for children requiring more than an initial OAE screening, the length of time over which the screening was completed ranged from 7 to 12 weeks. The median time required to complete a single OAE screening session was 4 minutes per child. The results demonstrate that OAE screening of young children using this protocol is practical and effective. The implications for conducting periodic hearing screening throughout early childhood are discussed.
\end{abstract}

(C) 2006 Elsevier Inc. All rights reserved.

Keywords: Screening; Hearing loss; Childhood

In the United States, approximately 1 out of every 300 children is born with a permanent hearing loss, making it the most common birth defect in the country. To put the incidence of congenital hearing loss in context, consider that in a representative sample of 10,000 newborns from the general population, 30 will have congenital hearing loss, but only 11 will have Down Syndrome, five will have Spina Bifida, and one will have phenylketonuria (NCHAM, 2005) Advances in technology have now made it possible to screen newborns for hearing loss and over the past 10 years the percentage of infants screened at birth has increased from 3 to $90 \%$ (White, 2004). While universal in concept, however, approximately $10 \%$ of newborns do not receive a hearing screening and in some states $50 \%$ or more of the infants who do not pass newborn hearing screening are lost to follow-up before receiving additional screening or diagnostic services (Centers for Disease Control, 2005). In addition, not all childhood hearing loss can be identified at birth because a child can lose his or her hearing at any point in early childhood. In fact, it is estimated that by school age approximately 3 out of every 300 students have a permanent sensorineural hearing loss (American Speech-Language-Hearing Association, 1993). In

\footnotetext{
* Corresponding author. Tel.: +1 3034995380 .

E-mail address: hearingheadstart@aol.com (W.D. Eiserman).
} 
addition to children who have a congenital or late-onset permanent hearing loss (occurring any time after birth), approximately $35 \%$ of preschoolers will have repeated episodes of ear infections that nearly always cause temporary hearing loss that can significantly disrupt language acquisition and educational progress (ASHA, 2004a). Hence, periodic hearing screening throughout early childhood is critical for identifying hearing-health conditions that can impede a child's development.

Each year Head Start provides services to more than 900,000 children with the mission to help children from low-income families start school ready to learn (Administration for Children and Families [ACF], 2005a). Head Start programs aim to address all areas of child development including early learning and literacy, health, disability services and family and community partnerships (ACF, 2005b). Reflecting the relationship between hearing, language acquisition, cognitive development, literacy, and school readiness, Head Start performance standards require that all children be screened for hearing loss within 45 calendar days of entering the program and that children not passing the screening be referred for diagnosis and intervention as needed (ACF, 2001). The emphasis being placed on helping children acquire pre-literacy and literacy skills as a part of the No Child Left Behind Act underscores the importance of identifying all children whose progress in these areas is likely to be compromised by the presence of an undetected hearing loss (The White House, 2002).

Current Head Start performance standards do not specify a particular hearing screening technique to be used. Rather, agencies are directed to consult with local content area experts and their Health Services Advisory Committees as they design and implement screening protocols (O'Brien, 2001). Unfortunately, many programs, local consultants, and/or advisory committees are not aware of available hearing screening procedures and equipment or the significant technological advances which have taken place during the past decade (Eiserman, 2003). For example, 53\% of hospitalbased screening programs are now using otoacoustic emissions (OAE) technology to conduct newborn screening (White, 2004).

OAE screening, when used with an appropriate protocol, also lends itself to screening children birth to 3 years of age because it is: (a) objective and not dependent on a behavioral response from the child (it can even be performed while the child is sleeping); (b) painless; (c) reliable and efficient (requiring about five minutes per child); (d) hand-held and portable (suitable for screening in either center or home-based settings); (e) simple to administer when a child initially enters an early childhood program, at annual intervals, and at any other time parents or educators have concerns about the child's hearing-health; and (f) straightforward to use and does not require technical skill or in-depth understanding of the auditory system.

Despite its suitability for screening the hearing of young children, OAE technology has seldom been used in early childhood screening programs like those implemented every day in Migrant, American Indian, and Early Head Start. Some Head Start programs attempt to satisfy their hearing screening requirements by referring to the child's newborn hearing screening results, even though those results reflect the hearing status of the child only at the time of birth and the results may be many months old at the point the child is entering the program. More commonly, Head Start staff members screen infants and toddlers for hearing loss by relying on physician reports indicating that ears had been "checked" (which is not equivalent to a physiologic hearing screening), by using subjective observations of the child's behavioral response to sound (such as hand clapping or bell ringing) or by collecting parent perceptions of the child's behavior (Munoz, 2003). Because hearing loss is an "invisible" condition, many infants and toddlers, especially those with moderate, mild, or unilateral hearing losses, do not exhibit observable symptoms of a loss until they are older. Prior to the use of physiologic screening tools, such as OAE screening, infants with hearing loss typically remained unidentified until $2 \frac{1}{2}$ years of age (Harrison, Roush, \& Wallace, 2003) - far too late for optimal language development. Children with mild or moderate losses have often not been identified until 4 years of age or until entering school (Commission on Education of the Deaf, 1988; Niskar, 1998).

\section{Objective}

When hearing loss goes undetected, the resulting language deficits can become overwhelming obstacles to literacy, educational achievement, socialization, and school readiness (Moeller, 2000). Physiologic screening has proven to be the most reliable, objective screening tool to identify young children at risk for hearing loss and subjective techniques can no longer be considered as viable hearing screening tools (Cunningham \& Cox, 2003). The purpose of this study was to examine the feasibility and benefits of assisting programs that serve infants and toddlers in transitioning from 
subjective hearing screening techniques to objective hearing screening practices that are now readily available. The study was designed to answer the following research questions:

1. What are the overall hearing-health outcomes and types of conditions identified for children 0-3 years of age when Migrant, American Indian, and Early Head Start staff members are trained to implement an OAE-based hearing screening and follow-up program?

2. How many children with permanent hearing loss are identified as a result of OAE screening?

3. How effective is a multi-step OAE hearing screening protocol in terms of:

(a) maximizing the identification of children having hearing-health conditions likely to interfere with normal hearing, while

(b) minimizing over-referral of children for medical or audiological follow-up who did not actually need treatment ("false-positive" referrals)?

4. How feasible is it for programs serving infants and toddlers to implement a multi-step screening and follow-up protocol?

\section{Method}

To evaluate the feasibility of implementing OAE hearing screening in Head Start programs, all Migrant, American Indian and Early Head Start programs serving children 0-3 years of age in Oregon, Utah, and Washington were provided with information describing the project and invited to participate. Programs electing to participate received screening equipment, training, resource materials, and ongoing technical support and audiological supervision. In turn, programs agreed to send staff to a 1-day training workshop and a subsequent 1-day follow-up training meeting, to submit OAE screening and follow-up data on all 0-3-year-old children in the program, and to communicate with the research team on a regular basis.

Prior to the initiation of this study, participating programs were screening for hearing loss by relying on physician reports indicating that ears had been "checked," by using the results from the child's newborn hearing screening test (which is indicative of hearing status only at the time the screening was conducted) and, more commonly, by using a variety of subjective hearing screening methods including parent questionnaires or a "startle test" involving ringing a bell or clapping hands behind a child. One of the programs had already purchased OAE equipment and was attempting to use it, but was not using an appropriate screening protocol. Another program had previously purchased an OAE device that was adequate for screening newborns, but was not well-designed for screening toddlers in natural settings. In interviews at the beginning of the study, staff in all of the programs expressed dissatisfaction with their current hearing screening methods, but reported that they had no means of making any improvement without appropriate guidance and assistance.

\subsection{Participants}

Staff from 52 sites (29 Migrant, three American Indian, and 20 Early Head Start sites) in Oregon, Utah, and Washington participated in the project. The 52 sites were diverse in many ways: 15 were in urban locations and 37 in rural locations; 40 served families primarily in center-based settings, two provided home-based services, and 10 served families in both center and home-based settings. Hence, participating programs included those where children were easily accessible in the center on a daily basis as well as home-based programs that extended across a 3000-squaremile area. While the American Indian and Early Head Start programs operated year-round, participating Migrant programs operated seasonal programs ranging from 6 to 9 months as well as some short-term programs ranging from 16 to 26 weeks. The mean number of children served by the participating programs was 165 , ranging from 38 to 982 .

From 2 to 13 staff members were trained in each site. Of the total screenings conducted on children, approximately $81 \%$ were performed by program staff with backgrounds in health and/or disabilities and $19 \%$ were performed by early childhood staff (including home visitors, and service coordinators). Table 1 summarizes the demographic backgrounds of the children who were screened in the participating programs. All children who were screened as a part of this project were between 0 and 3 years of age. 
Table 1

Summary of child demographics

\begin{tabular}{lc}
\hline Demographic variable & Number and Total percentage \\
\hline Gender & $1623(52 \%)$ \\
- Female & $1806(47 \%)$ \\
- Male & $57(1 \%)$ \\
- Unknown & \\
Age (months) Mean=22 S.D. =(13) & $941(27 \%)$ \\
- $0-12$ & $895(26 \%)$ \\
- $13-24$ & $1086(31 \%)$ \\
- 25-36 & $564(16 \%)$ \\
- 36-48 & \\
Type of program & \\
- Migrant Head Start & $2049(59 \%)$ \\
- Early Head Start & $1272(36 \%)$ \\
- American Indian Head Start & $165(5 \%)$ \\
Ethnicity & \\
- Hispanic & \\
- Caucasian & $2269(65 \%)$ \\
- American Indian & $727(21 \%)$ \\
- African American & $212(6 \%)$ \\
- Bi-racial & $87(2 \%)$ \\
- Asian & $53(2 \%)$ \\
\hline
\end{tabular}

\subsection{OAE hearing screening methodology}

Otoacoustic emissions testing is a non-invasive procedure that generates an objective response from the inner ear. During OAE screening, the screener places a small probe, fitted with a sensitive microphone, into the child's ear canal. The probe delivers a quiet tone or clicking sound into the ear. In a healthy ear, sound is transmitted through the middle ear to the inner ear where the outer hair cells of the cochlea respond by producing an emission sometimes described as an "echo." This emission travels back out through the middle ear and is then picked up by the microphone, analyzed by the screening unit, and a "pass" or "refer" result is displayed on the unit's screen. Every normal, healthy inner ear produces an emission that can be recorded in this way (Gorga et al., 1997). OAE screening can be performed in a variety of natural environments, including settings where other children are playing and vocalizing at a moderate level.

The ear will not pass the screening if there is: (a) a blockage in the ear canal; (b) a structural problem in the middle ear that interferes with hearing; (c) excess fluid present in the middle ear (often due to otitis media); or (d) a cochlea that is not responding normally to sound. Thus, OAE screening can help identify children who need to be evaluated for fluctuating hearing loss associated with otitis media or middle ear infection as well as children who have permanent hearing loss.

In an extensive review of large-scale studies examining the accuracy of OAE screenings with newborns, the Canadian Working Group of Childhood Hearing (2005) estimated sensitivity and specificity levels of just under 85 and $95 \%$, respectively. In a study of 1850 infants screened for hearing loss in Rhode Island, White et al. (1994) estimated sensitivity and specificity levels of 100 and 95\%, respectively. Smaller-scale studies have also examined OAE use with young children. In a study of 110 children ranging from 6 months to 15 years of age recovering from bacterial meningitis, Richardson, Williamson, Reid, Tarlow, and Rudd (1998) found that OAE was highly sensitive (100\%) and reasonably specific $(91 \%)$.

Based on the recommendations of six pediatric audiologists with extensive experience screening young children, and for purposes of uniformity in the study, a single brand of OAE equipment, the AuDX from Bio-logic Systems Corp (http://www.blsc.com/hearing/audx1.html), was used at all sites. The AuDX measures distortion product otoacoustic emissions with screening parameters set to deliver stimulus and measurement levels that are sensitive to hearing loss as low as 25 decibels (dB) hearing level (HL). If an emission is not present at these low levels, a refer result is obtained. 
While the screening is not diagnostic in nature, it is sensitive to mild to profound hearing loss and screens in the frequency (pitch or tone) range that is critical for normal speech and language development.

It is important to emphasize that OAE screening as performed in this study is not an audiological diagnosis of hearing status. OAE screening can, however, be performed by non-audiologists and is therefore an excellent first step in identifying children who may have a temporary or permanent hearing loss.

Staff at the Head Start locations participated in training workshops and multiple programs were typically trained during a workshop. A total of 112 people were trained with an average of 14 participants per training workshop. Training required approximately 6 hours to complete and included large-group instruction in which a training team of 3-4 people provided general information to the entire group of 12-16 trainees and small-group instruction in which each trainer assisted 3-4 screener trainees in learning to use the equipment by screening adults and children. Audiovisual and written materials were used during the training and were given to participants to ensure a standardized training approach and to give trainees materials that could be reviewed later.

\subsection{Hearing screening protocol}

A screening protocol was developed for use in early childhood programs that would be practical for screeners to follow and would also reflect accepted audiological and medical practices including current recommendations and guidelines of the Head Start performance standards. The protocol was designed to: (1) maximize the identification of children having permanent hearing loss as well as chronic middle ear disorders or other hearing-health conditions; and (2) minimize over-referral of children for medical or audiological follow-up who did not actually need treatment ("false-positive" referrals). Key components of the protocol included a visual inspection of the ear and up to three OAE screenings, if needed, ideally implemented over a 2-4 week period. Children not passing any of the OAE screenings were referred to a health care provider, and as needed, to a pediatric audiologist for evaluation. Although screeners were trained to follow the standard protocol, they were also instructed to exercise their own judgment when unusual circumstances (e.g., parent suspicion of hearing loss) warranted more immediate referral to a health care provider.

Key factors in monitoring the overall reliability of findings included screening equipment selection, protocol design, and data collection of individual child outcomes. Specifically, OAE screening technology was selected as the hearing screening method because: (1) no screener interpretation of results is required - screening outcomes are displayed in straightforward "pass" or "refer" terminology; and (2) studies cited previously have demonstrated that the technology has high sensitivity to hearing loss and a concomitantly low probability that screeners would obtain false negative results. Conversely, false positive results, which are linked to screeners' child management and probe fit skills, were minimized by employing a multi-step screening protocol and the availability of more than one trained screener at each site. Because the protocol required that children not passing the initial screening be screened twice more with OAE before being referred for evaluation, the protocol itself significantly limited false positive findings.

In addition, it is important to note that a "false positive" refer can also result from factors, such as "external" noise present in the environment or excess "internal" noise produced by movement or noise generated by the child. For this reason, the protocol suggested that most children not passing the initial OAE screening should be re-screened to minimize "false positive" referrals to health care providers.

Hearing screening outcome data were also reviewed regularly by the key contact at each site and by Project staff. If refer rates of a screener, or at a site, appeared unusually high, additional technical assistance was provided to improve screening accuracy. The data collection procedure itself, which tracked each child through the screening and diagnostic process, allowed researchers to evaluate the percentage of infants flagged by the OAE screening who were not ultimately identified as having a hearing loss or disorder. Thus, screening accuracy was largely controlled for by equipment capability and the protocol and data monitoring processes that required multiple screening and diagnostic procedures if a child did not pass the initial OAE screening.

\subsection{Data collection and dependent variables}

Screening forms were designed to collect child demographic and screening outcome information while guiding screeners through the screening protocol. Head Start staff used the hearing screening form, printed in triplicate, to communicate screening outcomes to the research team as well as to physicians or audiologists as needed. A diagnostic follow-up form was also created and used for the smaller subset of children who were referred from screening 
(approximately 5\% of the total sample). It allowed health care providers and audiologists within the communities where the children were served to document outcomes for Head Start staff and for researchers. Dependent variables are summarized below.

\subsubsection{Visual inspections}

This variable had two potential results for each ear: (1) pass, indicating no observable abnormalities, or; (2) refer, indicating an observable abnormality.

\subsubsection{OAE screening outcomes}

This variable had three potential results for each ear: (1) pass, indicating normal hearing; (2) can't test, indicating the child was not cooperative, generally because the child was distressed or would not sit quietly during the screening; or (3) refer, indicating possible hearing loss of cochlear origin or the presence of outer/middle ear blockage or fluid.

\subsubsection{Diagnostic outcome}

Diagnostic outcomes for children referred from either the visual or the OAE screening reflect the clinical judgment of various health care providers and audiologists in the communities where children were regularly served. These individuals did not receive training as a part of the study nor did the researchers attempt to control the diagnostic methods used.

\subsubsection{Health care provider evaluation outcomes}

When children not passing the OAE screening were referred to a health care provider, the provider checked for the presence of outer or middle ear disorders. Possible diagnoses included ear canal blockage, acute or chronic otitis media, non-patent PE tubes and other abnormalities of the outer or middle ear.

\subsubsection{Audiologist evaluation outcomes}

Children who were medically cleared by a health care provider and continued to refer on OAE screening were referred to an audiologist to evaluate hearing status. Possible diagnoses included: Middle ear disorder requiring further medical treatment; fluctuating conductive hearing loss (temporary hearing loss that improves or worsens depending upon the status of the middle ear); permanent conductive hearing loss; permanent sensorineural hearing loss; mixed hearing loss with both a conductive and sensorineural component; and other anatomical abnormalities of the peripheral and central auditory system.

In addition to the quantitative data collected from each participating program's hearing screening results, qualitative data were collected through notes and other written documentation accompanying screening and follow-up diagnostic forms, monthly interviews with staff at each participating program, reports by participants via email, telephone calls, or during group evaluation meetings about their successes, challenges and concerns about the OAE screening and follow-up program.

\section{Results}

As described earlier, four research questions were investigated as a part of this study. Results related to each of those questions are summarized below.

\subsection{Research question \#1: What are the overall hearing-health outcomes and types of conditions identified for children 0-3 years of age when Migrant, American Indian, and Early Head Start staff members are trained to implement an OAE-based hearing screening and follow-up program?}

Based on the multi-step screening protocol, of the original 3486 children who were screened, a total of 183 (5\%) were referred for medical or audiological follow-up. As shown in Table 2, of the 183 children referred for follow-up from screening, 80 were identified with a hearing loss or disorder requiring treatment or monitoring. A breakdown of these 80 cases revealed that six children were diagnosed with permanent hearing loss; 63 children were diagnosed with otitis media, which is almost always associated with fluctuating hearing loss (ASHA, 2004a; Gravel \& Wallace, 
Table 2

Summary of diagnostic outcomes

\begin{tabular}{ll}
\hline Diagnostic outcomes & Number of children \\
\hline Hearing loss or disorder requiring treatment or monitoring & $80(44 \%)$ \\
Permanent hearing loss & 6 \\
Otitis media (ear infection) & 63 \\
Occluded pressure equalization (PE) tubes & 2 \\
Excessive ear wax requiring removal & 7 \\
Congestion - decongestant recommended & 2 \\
No treatment recommended & $39(21 \%)$ \\
Exited before diagnosis completed/referral appointment completed & $64(35 \%)$ \\
& $n=183$ \\
\hline
\end{tabular}

2000); two children were treated for occluded pressure equalization tubes; and nine were treated for excessive earwax or congestion.

Of the 183 referred children, 39 were diagnosed as "normal" and no further treatment was recommended. It is not possible to determine whether these 39 cases, representing $1 \%$ of the total number of children screened, were initially "false positive" referrals or whether the children did indeed have a hearing problem at the time of the OAE screenings, such as a wax blockage in the outer ear or fluid in the middle ear, that was resolved prior to the diagnostic examination. Because most of the screeners in the study were trained only to perform the OAE screening, further examination of the ear canal and the tympanic membrane at time of the screening, through otoscopy or tympanometry, was not part of the suggested protocol. A total of 64 children who were referred from the OAE screening exited the Head Start program before medical or audiological referrals could be completed. Thus, diagnostic evaluations were available for 119 of the 183 referred children.

\subsection{Research question \#2: How many children with permanent hearing loss were identified as a result of OAE-based screening?}

As a result of the OAE screening and follow-up assessment, 6 out of the 3486 children in the Head Start programs were found to have a permanent hearing loss. Table 3 summarizes additional information about these six children, including type of hearing loss, the age at diagnosis, newborn screening results, along with background and current treatment information. Three of the children had a permanent sensorineural hearing loss (originating in the cochlea) while three had a permanent conductive loss (originating in the middle ear).

\subsection{Research question \#3: How effective was a multi-step OAE hearing screening protocol in terms of: (a) maximizing the identification of children having hearing-health conditions likely to interfere with normal hearing, while (b) minimizing over-referral of children for medical or audiological follow-up who did not actually need treatment ("false-positive" referrals)?}

Data on 3486 children screened during the 36-month project period were coded and analyzed to assess the outcomes of the multi-step screening and follow-up process to determine if the goals of the protocol were met. The first step in

Table 3

Information on children identified with permanent hearing loss

\begin{tabular}{lll}
\hline Case & Diagnosis and age at diagnosis & Newborn hearing screening results \\
\hline 1 & Bilateral, severe sensorineural loss and otitis media (9 months) & Referred; subsequently lost to follow-up \\
2 & Bilateral, severe conductive loss (30 months) & Passed \\
3 & Bilateral, mild/moderate conductive loss and otitis media (36 months) & Not screened at birth \\
4 & Bilateral, mild/moderate conductive loss (24 months) & Not born in U.S., not screened at birth \\
5 & Unilateral, mild/moderate sensorineural loss in left ear (15 months) & Passed \\
6 & Unilateral, mild/moderate high frequency sensorineural loss in left ear (24 months) & Passed \\
\hline
\end{tabular}




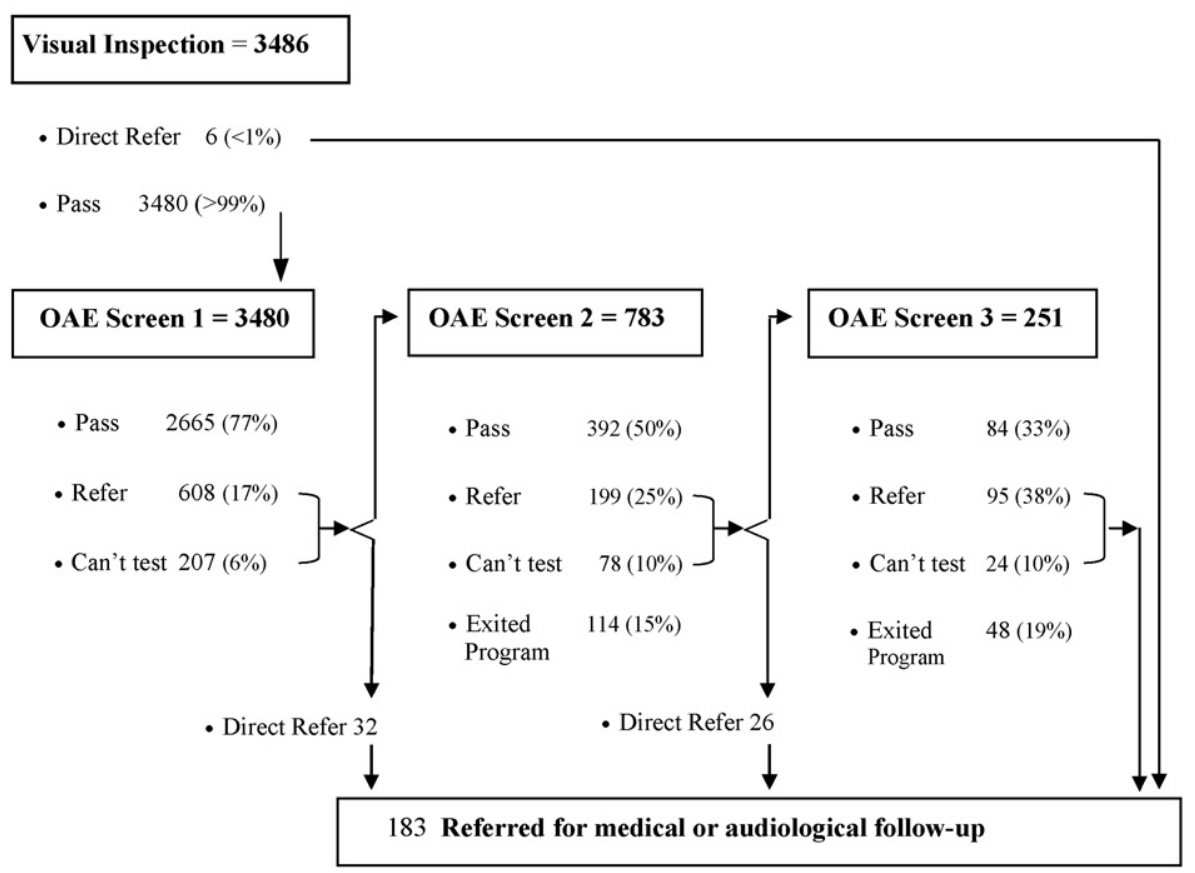

Fig. 1. Flow chart documenting screening results at each step in the hearing screening protocol.

the screening process was the visual inspection of the outer ear for abnormalities or obvious indicators of active ear infection. If anomalies were noted, children were referred directly for medical follow-up. As is shown in Fig. 1, a total of six children $(<1 \%$ of the children screened) were referred for medical follow-up from the visual inspection. The $3480(>99 \%)$ children passing the visual inspection were then screened using OAE equipment.

\subsubsection{OAE screen 1 outcomes}

Of the 3480 children receiving an initial OAE screening, 2665 (77\%) passed and required no further follow-up. A total of $608(17 \%)$ "failed" or "referred" on this first screening, while $207(6 \%)$ were documented as "can't test" (generally because children were uncooperative on that day). Thus, a total of 815 (23\%) children did not pass the initial OAE screening. Although the screening protocol suggested that children not passing the initial screening should typically be referred for a subsequent OAE screening before being referred for medical or audiological follow-up, screeners were instructed to exercise their own judgment in making a direct referral to a health care provider if circumstances warranted (e.g., if a child had a known history of ear infection, parents voiced concern about the child's hearing, the Head Start program would be closing in the near future before subsequent screening could be performed, etc.) Thus, of the 815 children who did not pass this initial OAE screening, 32 were referred directly to a health care provider on the basis of these types of additional concerns.

\subsubsection{OAE screen 2 outcomes}

Of the 783 children needing a second screening, 392 (50\%) passed and required no further follow-up. A total of 199 $(25 \%)$ "failed" or "referred" on the screening and $78(10 \%)$ could not be tested. The remaining 114 (15\%), however, did not receive this second screening due to the program closing for the year/season or the child exiting the Head Start program. Of the 277 children who did not pass this second OAE screening, 26 (9\%) were referred directly to a health care provider due to additional concerns. Thus, in accordance with the three-step screening protocol, a total of 251 children were expected to receive an OAE screen 3.

\subsubsection{OAE screen 3 outcomes}

Of the 251 children needing a third screening, 84 (34\%) passed and required no further follow-up. A total of 95 $(38 \%)$ "failed" or "referred" on the screening and $24(10 \%)$ could not be tested. The remaining 48 (19\%) however, 
did not receive this third screening due to the program closing for the year/season or the child exiting the Head Start program. Thus, in accordance with the three-step screening protocol, 119 children were referred for medical follow-up from the third screening.

\subsubsection{Medical or audiological follow-up outcomes}

Including the children who had been referred from the visual inspection (6), directly after OAE screen $1(n=32)$ or directly after OAE screen $2(n=26)$, as well as those not passing after completing all three steps of the screening process $(n=119)$, a total of 183 children were referred for medical or audiological follow-up. This represents a referral rate of only $5 \%$ of the total 3486 children in the study. As described previously in Table 2, 80 (44\%) of the 183 children were found to have a hearing loss or disorder while $39(21 \%)$ were determined to have "normal" hearing. The remaining 64 children (35\%) exited the Head Start program, or the program closed, before the follow-up could be conducted.

Taken together, these data can be used to calculate the specificity of the three-step screening protocol used in this study. Of the 3486 children who were screened, 3260 either passed the screen $(n=3141)$ or had evaluation data available at the completion of the process. Of the 119 children with evaluation data, 39 had normal hearing and 80 had a hearing problem. Following standard definitions for evaluating screening tests (Greenhalgh, 1997), there were 80 true positives (2.5\%), 39 false positives (1.2\%), and 3140 true negatives (96.3\%); the specificity of the screening test (true negatives/[true negatives + false positives]) was $98.8 \%$, and the positive predictive value (true positives/[true positives + false positives]) was $67.2 \%$.

\subsection{Research question \#4: How feasible is it for programs to implement a multi-step screening and follow-up protocol?}

To further evaluate the practicality of doing OAE-based hearing screening in early childhood programs, screeners were asked to note the approximate amount of time required to conduct each screening session which included preparing the individual child, conducting the screening, and documenting the screening results for that child. The median time was 4.0 minutes (mean of 4.8 minutes and a range of 1-30 minutes). None of the programs reported that they needed to hire additional staff or increase work hours for existing staff to complete the OAE screenings.

An analysis of the dates at which first, second, and third OAE screenings were done revealed that screeners were not always successful in completing the screening during the time frame suggested by the protocol. The protocol stated that if subsequent screening was needed for a child, it should be performed within 2 weeks of the previous attempt. Thus, the entire three-step screening protocol should have been completed within a 30-day time frame. Instead, the average length of time elapsing between OAE screen 1 and OAE screen 2 for the 669 affected children was 49 days. The average length of time between OAE screen 2 and OAE screen 3 for the 203 affected children was 35 days. Interviews with Head Start staff suggested that a number of factors contributed to the time lag between screenings, including competing demands on staff time at the beginning of the program year and the significant distance staff sometimes had to travel to screen or rescreen children in home-based programs. Finally, staff may not have understood the importance of conducting the rescreens in a timely way to maximize the effectiveness of the screening process.

\section{Discussion}

The dramatic improvements in hearing screening technology, the steady growth of hospital-based newborn hearing screening programs, and the concurrent emergence of state Early Hearing Detection and Intervention (EHDI) programs over the past decade hold important implications for improving hearing screening practices in early childhood programs. This study showed that Head Start staff members from a variety of backgrounds were able to use OAE equipment to efficiently complete physiological hearing screening of infants and toddlers when provided with comprehensive training, including "hands-on" screening experience, a structured, appropriate protocol to follow, forms for recording results and tracking children who needed further follow-up, and ongoing support and monitoring from a pediatric audiologist.

Overall, Head Start staff members were able to successfully identify children who were ultimately diagnosed with a wide range of hearing-health conditions warranting monitoring and treatment. The overall referral rate of 5\% also compares favorably with the referral rates reported by effective hospital-based newborn hearing screening programs 
(White, 2003). Additionally, the specificity of $98.8 \%$ and the positive predictive value of $67.2 \%$ demonstrates that "over-referral" was not a problem.

As a result of the OAE screening conducted in this study, six children with permanent hearing loss were identified who were either not screened at birth, were screened and subsequently lost to follow-up after hospital discharge, or who passed the newborn hearing screen but presented with a hearing loss at some point during their early childhood years. As a result of the identification through screening and follow-up, these children were connected with audiological services, local early intervention services, and family support programs.

In understanding the implications of these findings, it is helpful to remember that the number of children with hearing loss that newborn hearing screening programs expect to identify at birth is approximately 3 per 1000 (National Center for Hearing Assessment and Management, 2005). Although the number of children screened in this study is not a large enough sample to draw firm conclusions about prevalence rates, these data suggest that two additional children per 1000 with permanent hearing loss could be identified by conducting OAE-based hearing screening in Head Start programs. Screening in other early childhood programs would likely increase this number even more.

Several additional implications related to cases shown on Table 3 merit further discussion. Case \#1 serves as an example of how periodic hearing screening throughout early childhood can help to identify children who refer from the newborn hearing screening and are lost to follow-up before assessment and intervention could be provided. It is important to note that the number of children lost to follow-up from newborn hearing screening is significant - some states report that as many as $50 \%$ of infants who need follow-up after newborn hearing screening may not be receiving it in a timely way or at all (CDC, 2005).

Cases \#2, \#5, and \#6 demonstrate that periodic hearing screening during early childhood is also vital for identifying children whose hearing loss likely originated after the newborn period (often described as a "late onset" loss). Despite passing the hearing screening at birth, by 15 to 30 months of age these children referred on the OAE screening. Although their hearing loss at the point of diagnosis was considered to be mild/moderate, and in two cases unilateral, it is important to remember that hearing loss among young children is often a progressive condition, worsening over time (Smith, Bale, \& White, 2005). The implication of early identification in these cases is that the children can be provided with additional services during the critical language-learning years and also be monitored closely to modify the treatment as necessary.

Finally, Cases \#3 and \#4 illustrate that periodic screening is important since some children were never screened at birth because they were born at home, were not born at hospitals performing newborn hearing screening, were discharged from the hospital without being screened, or were born in a country not doing universal newborn hearing screening. It is not known whether the hearing loss of these children was congenital or presented during early childhood. However, had the loss remained unidentified, these children would have likely experienced language delays and academic underachievement (Moeller, 2000).

The value of using the OAE equipment to screen children for permanent hearing loss after fluctuating middle ear conditions have been resolved is also highlighted by these cases, because two of the six children were diagnosed as having a "mixed" hearing loss that included both otitis media and a permanent hearing loss. Early education program providers often assume, incorrectly, that if the child is referred to a health care provider that all "ear" or "hearing" disorders will be identified and treated. The reality is that while most health care providers are familiar with treating middle ear infection, very few have the capacity to objectively screen children for a permanent hearing loss.

Finally, of the six children identified with permanent hearing loss, four were diagnosed as having a bilateral loss (both ears) while two had a unilateral loss (one ear). Although the advantage of identifying a bilateral hearing loss is widely understood, the significance of identifying unilateral hearing loss tends to be under-appreciated. An assumption is often made that a hearing loss in one ear can be easily compensated for by the other ear. Because children with unilateral hearing loss often respond to sound in a way that would indicate they are hearing, particularly in one-on-one interactions, a unilateral loss is especially difficult to identify using subjective methods. Furthermore, although children with unilateral hearing loss, or even mild bilateral loss, may be able to compensate for that loss in some contexts, their ability to discriminate speech sounds in an environment where competing background noise is present is often compromised. This is why unilateral or mild hearing loss often goes unidentified until children are in school and language and academic delays become apparent (ASHA, 2004b; Bess, Dodd-Murphy, \& Parker, 1998). Unilateral hearing loss can also be associated with progressive loss that may eventually affect the other ear. Therefore, identifying both bilateral and unilateral losses is important because both can have a significant negative effect on language acquisition, socialization, and academic development. 
In addition to the six children identified with permanent hearing loss, 74 other children in the study were diagnosed with a hearing problem, of whom 63 were diagnosed with otitis media and two were being treated for otitis media who had occluded pressure equalization tubes that were not functioning as intended. Although there is lively professional debate on the effect that otitis media may have on language development and on what constitutes optimal intervention (Roberts et al., 2004; Vernon-Feagans \& Manlove, 2005), the OAE hearing screening process was valuable in helping health care providers identify children with compromised hearing-health who needed further monitoring or treatment.

Unfortunately, a total of 64 children who were referred from the OAE screening exited the Head Start program before a diagnostic evaluation could be completed making it impossible to determine the hearing status for these children. Of the 119 referred children who did receive a diagnosis however, 80 (67\%) were found to have a hearing loss or disorder. Using the same percentages to extrapolate to the 64 children who exited the program, it is probable that approximately 42 of the 64 had a hearing loss or disorder and that approximately 3 of those 64 may have had a permanent hearing loss that remained unidentified. This extrapolation suggests that if programs were able complete follow-up for all referred children in a timely way, the incidence of children identified with hearing-health conditions in Head Start may be even greater than the findings presented in this study.

Several questions remain related to refining the hearing screening protocol in order to maximize the number of children appropriately referred for follow-up while minimizing false positive referrals and the number of children who exit the program before screening or diagnosis can be completed. It is clear that a protocol prescribing more than a single OAE screening is helpful in minimizing "false positive" referrals. In the present study, if a one-step OAE screening process had been used, all 815 children not passing the initial screening would have been referred which would likely represent unnecessary referrals to health care providers. Using a multi-step protocol, however, of the 783 children who were referred for an OAE screen 2, $392(50 \%)$ passed on that second screening. At this point in the screening process, a total of 3057 (88\%) of the original 3486 children had passed the OAE screening which represents an $11 \%$ increase from the $77 \%$ passing the OAE screen 1 . It would therefore appear that adding a second screening step is very helpful in minimizing "false-positive" referrals because children who were uncooperative and could not be tested, or who referred on OAE 1, possibly due to screener error or temporary congestion associated with head-colds, passed the OAE screen 2. A less positive aspect of the multi-step screening process, however, was that over that same time period, 114 children, representing approximately $14.6 \%$ of those referred after the first OAE screen, did not receive the second screening because they had exited the program or the program had closed.

In the final screening step, 84 of the 251 children referred for OAE screen 3, passed. At this point in the screening process, a total of $3141(90 \%)$ of the original children had passed the OAE screening which represents only a $2 \%$ increase in the overall pass rate from OAE screen 2. In the same time frame, an additional 48 children exited the program before OAE screen 3 could be completed.

The analysis raises questions about the relative advantages and disadvantages of implementing a two-step, rather than a three-step, OAE screening protocol. Advantages of a two-step screening process potentially include: (1) decreasing the number of children who exit the program before diagnosis can be made and treatment initiated, and (2) decreasing the length of time it takes for programs to complete the screening and make a referral. In this study, 48 children who had failed the first two steps of the screening protocol exited the program before receiving a third screening and an additional 64 exited after the third screening, but before an evaluation was completed. It is highly likely that some of these children had a permanent or fluctuating hearing loss that remained unidentified. The objective of the multi-step screening protocol was to allow 7-14 days to elapse between screenings so that minor head-cold congestion could be resolved while more serious middle ear conditions, which would be unlikely to resolve spontaneously within the total 4-week period, would be identified. In many instances, the time elapsing between screenings was much longer than desired. Children who were referred to a health care provider from the multi-step OAE screening, but were diagnosed by the provider as "normal" may not represent "false positive" screening results. These referrals may instead represent cases where fluctuating middle ear conditions existed at the time of screening, but were never documented in the child's medical records. This would be an unfortunate outcome since each occurrence of otitis media needs to be documented, even if not treated initially. This allows health care providers to monitor the condition and counsel parents on adaptations that can be made to accommodate any temporary hearing loss that may be occurring (Rosenfeld et al., 2004). The unintended effects of an extended screening protocol were probably exacerbated by the fact that not all children were seen promptly by a provider even after a referral was made. No child-specific data was collected to assess the amount of time elapsing after a referral was made before the child was seen by a provider, but anecdotal data from staff suggested that it could be challenging to get children assessed by providers in a timely way. 
Conversely, the disadvantages of a two-step screening protocol, rather than a three-step protocol, may include an increased number of "false positive" referrals to health care providers. Most OAE-based newborn hearing screening programs in hospitals use a two-step protocol. One significant difference, however, is that toddlers are more difficult to screen than infants because of their increased physical activity levels, therefore screeners are likely to generate more "false positive" results. In the present study, if the third step of the OAE screening had been eliminated, 319 children, rather than 183, would have been referred for follow-up, representing a 9\%, rather than a 5\%, referral rate. This would be acceptable if the majority of children were found to have conditions requiring monitoring or treatment, but would represent an overly high referral rate if no treatable conditions were identified. Further study will be necessary to determine whether a two- or three-step protocol creates the best balance in minimizing the "false positive" refer rate while maximizing the number of children who are seen by a provider in a timely way.

Finally, the potential for expediting referrals to a health care provider when the individual performing the OAE screening is also qualified to perform screening specific to middle ear disorders merits further consideration and exploration. Because most of the screeners in the study were not nurses and were trained only to perform the OAE screening, further examination of the ear canal and the tympanic membrane through otoscopy at time of the screening was not part of the suggested protocol. Likewise, neither tympanometry nor reflectometry were available and screeners were therefore not trained to attempt any further screening of the middle ear. If individuals conducting the OAE screening were also qualified to perform a more in-depth visual screen of the ear canal and the tympanic membrane, or to conduct tympanometry or reflectometry, it might be possible to refer children to health care providers more efficiently.

If Head Start and other early childhood programs are to help children start school ready to learn, it is critical that any barriers to learning be identified as early as possible. National programs like Medicaid's Early and periodic screening, diagnostic and treatment (EPSDT) service and Part C of the Individuals with Disabilities Education Act are also committed to providing hearing screenings for young children; however, subjective methods are still typically used in these programs as well. The close connection between hearing, language acquisition, literacy and school readiness demands that as technology improves, so also should the quality of hearing screening during the early childhood period. The results of this study demonstrate that OAE technology, when used with an appropriate screening and follow-up protocol, can make a valuable contribution in helping to identify a wide range of hearing-health conditions that can potentially disrupt language acquisition, literacy, socialization and overall school readiness.

\section{Acknowledgments}

The study reported in this article was funded in part by the Administration for Children and Families, Head Start Bureau and the Maternal and Child Health Bureau under Grant 6 H61 MC 00006-02-02 to the National Center for Hearing Assessment and Management at Utah State University. The opinions expressed in the article are those of the authors and do not represent the official position of any of the funding agencies. The findings of this study should in no way be construed as an endorsement of any particular manufacturer of hearing screening equipment by the authors, their institutions or by the funding agencies. The authors wish to express their appreciation for the assistance provided to this project by Karen Munoz, Jim O'Brien, Sherry Johnson and the many Head Start staff members who participated in the study.

\section{References}

Administration for Children and Families (ACF) (2001). Performance Standard 1204.20 - Child health and developmental services. Retrieved April 6, 2005 from http://www.acf.hhs.gov/programs/hsb/performance/130420PS.html.

Administration for Children and Families (ACF) (2005a). Head Start Bureau. Retrieved April 6, 2005 from http://www.acf.hhs.gov/opa/fact_sheets/ headstart_factsheet.html.

Administration for Children and Families (ACF) (2005b). Head Start program fact sheet. Retrieved April 6, 2005 from http://www.acf. hhs.gov/programs/hsb/research/2005.html.

American Speech-Language-Hearing Association. (1993). Guidelines for audiology services in the schools. ASHA, 35(Suppl. 10), $24-32$.

American Speech-Language-Hearing Association (2004a). Causes of hearing loss in children. Retrieved April 6, 2005 from http://www.asha. $\mathrm{org} /$ public/hearing/disorders/causes.html.

American Speech-Language-Hearing Association (2004b). Even minimal, undetected hearing loss hurts academic performance, research shows. Retrieved April 6, 2005 from http://www.asha.org/about/news/releases/2004/04ConvMinHrngLoss.html. 
Bess, F. H., Dodd-Murphy, J., \& Parker, R. A. (1998). Children with minimal sensorineural hearing loss: Prevalence, educational performance, and functional status. Ear and Hearing, 19, 339-354.

Canadian Working Group on Childhood Hearing (2005). Early hearing and communication development: Canadian Working Group on Childhood Hearing $(\mathrm{CWGCH})$ resource document. Ottawa, Canada: Minister of Public Works and Government Services.

Centers for Disease Control (2005). Infants tested for hearing loss - United States, 1999-2001. Retrieved May 5, 2005 from http://www.cdc.gov/ ncbddd/ehdi/documents/mm5241.pdf.

Commission on Education of the Deaf. (1988). Toward equality: Education of the deaf. Washington, DC: Author.

Cunningham, M., \& Cox, E. O. (2003). The committee on practice and ambulatory medicine and the section on otolaryngology and bronchoesophagology. Hearing assessment in infants and children: Recommendations beyond neonatal screening. American Academy of Pediatrics, 111, 436-440.

Eiserman, W. (2003). Getting a hearing head start. Presented at the National Zero to Three Conference. Washington, DC.

Gorga, M. P., Neely, S. T., Ohlrich, B., Hoover, B., Redner, J., \& Peters, J. (1997). From laboratory to clinic: A large scale study of distortion product otoacoustic emissions in ears with normal hearing and ears with hearing loss. Ear and Hearing, 18, 440-455.

Gravel, J. S., \& Wallace, I. F. (2000). Effects of otitis media with effusion on hearing in the first 3 years of life. Journal of Speech and Hearing Research, 43, 644-651.

Greenhalgh, T. (1997). How to read a paper: Papers that report diagnostic or screening tests. British Medical Journal, 315, 540-543.

Harrison, M., Roush, J., \& Wallace, J. (2003). Trends in age of identification and intervention in young children with hearing loss. Ear and Hearing, $24,89-95$.

Moeller, M. P. (2000). Early intervention and language development in children who are deaf and hard of hearing. Pediatrics, $106(3)$, E43.

Munoz, K. (2003). Survey of current hearing screening practices in Early Head Start, American Indian Head Start and Migrant Head Start programs. National Center for Hearing Assessment and Management, Utah State University.

National Center for Hearing Assessment and Management (2005). Universal newborn hearing screening: Fact sheet. Retrieved May 5, 2005 from http://www.infanthearing.org/resources/fact.pdf.

Niskar, A. S. (1998). Prevalence of hearing loss among children 6 to 19 years of age: The third national health and nutritional health examination survey. Journal of the American Medical Association, 279, 1071-1075.

O'Brien, J. (2001). How screening and assessment practices support quality disabilities services in Head Start, Head Start Bulletin: Enhancing Head Start Communication, U.S. Department of Health and Human Services, Administration for Children and Families, Administration for Children, Youth and Families, Head Start Bureau, April, No 70.

Richardson, M. P., Williamson, T. J., Reid, A., Tarlow, M. J., \& Rudd, P. T. (1998). Otoacoustic emissions as a screening test for hearing impairment in children recovering from acute bacterial meningitis. Pediatrics, 102(6), 1364-1368.

Roberts, J., Hunter, L., Gravel, J., Rosenfeld, R., Berman, S., Haggard, M., et al. (2004). Otitis media, hearing loss, and language learning: Controversies and current research. Journal of Developmental and Behavioral Pediatrics, 25, 110-122.

Rosenfeld, R., Colpepper, L., Doyle, K., Grundfast, K., Hoberman, A., Kenna, M., et al. (2004). Clinical practice guideline: Otitis media with effusion. Otolaryngology-Head and Neck Surgery, 130(Suppl.), 95-118.

Smith, R. J., Bale, J. F., \& White, K. R. (2005 March 5). Sensorineural hearing loss in children. Lancet, 365, 879-890.

The White House (2002). Good start, grow smart: The Bush administration's early childhood initiative. Retrieved April 6, 2005 from http://www.whitehouse.gov/infocus/earlychildhood/sect1.html.

Vernon-Feagans, L., \& Manlove, E. E. (2005). Otitis media, the quality of child care, and the social/communicative behavior of toddlers: A replication and extension. Early Childhood Research Quarterly, 20, 306-328.

White, K. R. (2003). The current status of EHDI programs in the United States. Mental Retardation and Developmental Disabilities Research Reviews, 9(2), 79-88.

White, K. R. (2004). Early hearing detection and intervention programs: Opportunities for genetic services. American Journal of Medical Genetics, 130(A), 29-36.

White, K. R., Vohr, B. R., Maxon, A. B., Behrens, T. R., McPherson, M. G., \& Mauk, G. W. (1994). Screening all newborns for hearing loss using transient evoked otoacoustic emissions. International Journal of Pediatric Otorhinolaryngology, 29, 203-217. 